\title{
What is the bed availability gap underlying chronic emergency department access block?
}

\author{
Peter A. Cameron, MBBS, MD*
}

All emergency care systems in the developed world suffer from chronic access block to inpatient beds. There have been countless studies, commentary, and policies over the last few decades, describing initiatives to solve this seemingly insurmountable problem. In this issue of the journal, Innes and colleagues discuss a fundamental concept underpinning solutions to emergency department (ED) access block and overcrowding. ${ }^{1}$ They believe that the "gap" between a dysfunctional, unsafe, unworkable ED and a safe ED working environment is about one percent of the total operating capacity of typical large urban hospitals. Improving hospital operational efficiency and effectiveness by a very small amount could resolve all of our problems in the ED. I like the concept!

So, why is everyone so slow to sort this out, why don't we cancel one percent of electives, introduce flexible wards that give us "surge" capacity, ask our colleagues on the inpatient team to work one percent faster, discharge patients a few hours earlier? After all, everyone agrees that $\mathrm{ED}$ crowding due to inpatient boarding is unsafe; is associated with excess deaths, complications, and morbidity; decreases hospital efficiency; and makes the lives of emergency clinicians difficult, resulting in dissatisfaction and burnout. This has been known for more than 10 years. $^{2}$ Is the world mad, and has the world just simply overlooked an easy fix?

The strange thing is that, for the last 30 years that I have been working in this space, every initiative to change that one percent gap has only resulted in temporary improvements at the institution and rarely resulted in system-wide improvements. Many of the initiatives have certainly enabled better, more patient-centred care; however, the initiatives have not necessarily improved access block indicators. There are many examples, including short stay units, to get people sorted quickly, care coordinators to enable safe discharge, fast investigation turnarounds, senior decision-making at the front door, timely inpatient ward rounds, and community outreach programs to avoid hospital presentation. Many of the initiatives reflect changes in community expectations in the availability of services and technological advancements in healthcare delivery. Yet, despite the literally hundreds of initiatives, we still have a situation where Canada, one of the wealthiest and most progressive countries in the world, has the sickest and most vulnerable emergency patients routinely waiting more than 24 hours for an inpatient bed in a dangerous and unsuitable environment for inpatient care. In fact, wait times for inpatient beds are increasing, and more than $10 \%$ of patients are waiting more than 30 hours to be in a safe ward environment. ${ }^{3}$

I am sure that all of the improvements in emergency care that have been undertaken in Canada to alleviate access block over the last decade would have freed up more than one percent of operational capacity. It is apparent that just like opening a new hospital or opening a new freeway, providing additional capacity, will always increase demand - or possibly allow previously unidentified demand to be met. This is more likely to be the case in a system where services are free or highly subsidized, and thus the price signal is limited. The presumption is that there is almost unlimited demand for health services. The "brake" on emergency service provision is the ED queue. This is very similar to elective surgery, where elective waiting times are used to limit demand and ensure that patients are prioritized (hopefully) according to urgency and need. Queuing can be either orderly or disorderly and unsafe.

From the *Emergency and Trauma Centre, The Alfred Hospital, Melbourne, Victoria, Australia.

Correspondence to: Professor Peter A. Cameron, Academic Director Emergency and Trauma Centre, The Alfred Hospital, Melbourne, Australia 3000; Email: peter.cameron@monash.edu

(C) Canadian Association of Emergency Physicians

CJEM 2019;21(2):165-166

DOI 10.1017/cem.2019.5 
Despite the gloom and despondency of many of my colleagues, sometimes resulting in helpless acceptance of the status quo, I think we should use the information provided in the Innes article to prosecute change, advocate for our patients, and demand a safe environment for patients and staff. Public hospitals and hospital systems will always have a mismatch between demand and service provision. This is even the case when there are high levels of public expenditure. Increased bed numbers and service provision can result in temporary reprieve but, inevitably, increases in capacity become overwhelmed by demand. It is never possible to "solve" the problem of emergency overcrowding or elective waiting lists. It is only possible to manage them safely.

The facts are, according to Innes, that the difference between a "safe" and "unsafe" ED is a tiny percentage of operational capacity. This debate is about hospital operational directors having tight control of patient flow across the hospital system with safety signals to ensure that dangerous practices, such as patients boarding in ED for more than 24 hours, don't happen. This is the equivalent of ED policies such as only performing procedural sedation in a fully equipped resuscitation bay or operating theatres not opening without a surgeon.

Clearly, much more work needs to be done to ensure that health service operational directors have the appropriate tools and policies to manage a complex and dynamic situation, where emergency demand can fluctuate hour by hour. The current operational model of managing hospitals at close to $100 \%$ or more of maximum capacity does not work. There are now many publications and policy documents to help us with this. A starting point for hospitals and health policymakers and funders must be that certain practices are unacceptable and unsafe and that the hospital administration is accountable for adverse events related to poor operational procedures. Patients staying more than 24 hours should be a "never" event. Patients waiting on ambulance trolleys due to a lack of available beds should not occur.

If we can manage access block with a one percent change in operational and organizational efficiency, I think we owe it to our patients and our specialty to deliver.

Keywords: Emergency Department, crowding, bed block

Competing interests: None declared.

\section{REFERENCES}

1. Innes GD, Sivilotti MLA, Ovens H, et al. Emergency overcrowding and access block: A smaller problem than we think. C7EM 2019;21(2):177-185.

2. Cameron PA, Joseph AP, McCarthy SM. Access block can be managed. Med 7 Aust 2009;190 (7):364-8.

3. Canadian Institute for Health Information. Emergency department wait times continue to rise; 2018. Available at: https://www.cihi.ca/en/emergency-department-wait-times-incanada-continuing-to-rise (accessed December 17, 2018). 